\title{
Impact of baseline steroids on efficacy of programmed cell death-1 (PD-1) and programmed death-ligand 1 (PD-L1) blockade in patients with advanced non-small cell lung cancer
}

\author{
Maria Jove $^{1,2 \#}$, Noelia Vilariño ${ }^{1,2 \#}$, Ernest Nadal $^{1,2}$ \\ ${ }^{1}$ Department of Medical Oncology, Catalan Institute of Oncology, L'Hospitalet (Barcelona), Spain; ${ }^{2}$ Research in Solid Tumors (CReST) Group, \\ Oncobell Program, Bellvitge Biomedical Research Institute, L'Hospitalet (Barcelona), Spain \\ \#These authors contributed equally to this work. \\ Correspondence to: Dr. Ernest Nadal. Department of Medical Oncology, Catalan Institute of Oncology, Avda Gran Via 199-203, L'Hospitalet \\ (Barcelona), Spain. Email: esnadal@iconcologia.net. \\ Provenance: This is an invited article commissioned by the Section Editor Hengrui Liang (Department of Thoracic Surgery, Guangzhou Medical \\ University, Guangzhou, China). \\ Comment on: Arbour KC, Mezquita L, Long N, et al. Impact of Baseline Steroids on Efficacy of Programmed Cell Death-1 and Programmed Death- \\ Ligand 1 Blockade in Patients With Non-Small-Cell Lung Cancer. J Clin Oncol 2018;36:2872-8.
}

Submitted May 01, 2019. Accepted for publication Jun 14, 2019.

doi: $10.21037 /$ tlcr.2019.06.06

View this article at: http://dx.doi.org/10.21037/tlcr.2019.06.06

Immune checkpoint inhibitors (ICI) have become part of the standard of care of patients with locally advanced and advanced non-small cell lung cancer (NSCLC) (1). Corticosteroids are broadly used as premedication for most chemotherapy regimens and are frequently used to alleviate pain or dyspnea, to stimulate appetite, or to palliate symptoms associated with brain or epidural metastases. However, corticosteroids have anti-inflammatory and immunosuppressive effects that act over both innate and adaptive immunity. Based on this, patients treated with corticosteroids at doses equal to or higher than $10 \mathrm{mg} / \mathrm{day}$ of prednisone or equivalent have been systematically excluded from clinical trials of ICI.

Corticosteroids are characterized by their pleiotropic effect on multiple signaling pathways, including the modulation of the immune system. In cancer, glucocorticoids have an impact along all immune-cycle canonical steps: from the release of cancer cell antigens to lymphocyte trafficking within the tumor as well as in the effector phase of tumor destruction (2). Corticosteroids suppress the initial activation of inflammatory pathways that are involved in the detection of noxious agents, such as toll-like receptor (TLR) signaling and the NF- $\mathrm{KB}$ pathway. Corticosteroids reduce eicosanoid and prostaglandin production and inhibit the expression of pro-inflammatory cytokines like IL-1 $\alpha$, IL-1 $\beta$, IL-2, IL-6,
IL-12, interferon- $\gamma$ (IFN- $\gamma$ ), tumor necrosis factor (TNF), and granulocyte-macrophage colony-stimulating factor (GM CSF). Moreover, glucocorticoids reduce lymphocyte extravasation by inhibiting endothelial expression of E-selectin and integrin ligands [vascular cell adhesion molecule 1 (VCAM-1); intercellular adhesion molecule 1 (ICAM-1)] and decrease the secretion of chemoattractants and chemokines (CXCL8 and CCL2), and leucocyte adhesion molecules (CD44 and integrins) in the tumor microenvironment. Finally, corticosteroids impair activation of $\mathrm{T}$ lymphocytes, by blocking $\mathrm{T}$ helper 1 and recruiting $\mathrm{T}$ regulatory cells and can also induce M2 macrophages polarization.

Corticosteroids also lead to changes in the peripheral blood immune cells. In a recent study, Fucà et al. showed that early use of steroids was significantly correlated with higher median absolute neutrophil count, neutrophil to lymphocyte ratio (NLR), and derived NLR (dNLR) with a lower median absolute and relative eosinophil count (REC) after ICI treatment (3). A high NLR/dNLR and a low REC at 4 and 6 weeks after treatment were associated with reduced benefit from ICI treatment suggesting that early use of steroids may worsen patient outcome by modulating peripheral white blood cells.

In a study recently published in Fournal of Clinical 
Oncology, Arbour et al., retrospectively evaluated the impact of baseline steroids on the efficacy of programmed cell death-1 and programmed death-ligand 1 [PD-(L)1] blockade in patients with NSCLC (4). As corticosteroids are often used in patients with NSCLC to control tumor-related symptoms or chronic obstructive pulmonary disease (COPD) exacerbations, it is highly clinically relevant in this setting to know whether concomitant use of corticosteroids may have a detrimental effect. This study included 640 patients with NSCLC treated with single PD-(L)1 blockade from Memorial Sloan Kettering Cancer Center (MSKCC) and Institute Gustave Roussy (IGR). Ninety patients (14\%) were treated with baseline steroids $\geq 10 \mathrm{mg} /$ day at the initiation of PDL-(L)1 blockade (53 patients in the MSKCC cohort and 37 in the IGR cohort). The clinicopathologic characteristics were well balanced between patients who received or did not receive corticosteroids, with the exception of history of brain metastases and poor Eastern Cooperative Oncology Group (ECOG) performance status (PS), which were more common in the group receiving corticosteroids at baseline. In both cohorts, patients receiving steroids at baseline had statistically shorter median progression-free survival (PFS) (1.9 versus 2.6 months in the MSKCC cohort, $\mathrm{P}<0.001$ and 1.7 versus 1.8 months for the IGR cohort, $\mathrm{P}<0.001)$ and shorter median overall survival (OS) (5.4 versus 12.1 months for the MSKCC cohort, $\mathrm{P}<0.001$ and 3.3 versus 9.4 months for the IGR cohort, $\mathrm{P}<0.001)$. In the multivariate analysis of the pooled cohort of MSKCC and IGR the use of baseline corticosteroids at $>10 \mathrm{mg} /$ day of prednisone or equivalent was independently associated with worse PFS and OS, after adjusting for smoking history, ECOG PS, and history of brain metastases.

The authors also analyzed the effect of different corticosteroid doses and observed that the detrimental effect was similar in patients treated with $10-19 \mathrm{mg} /$ day or $>20 \mathrm{mg} /$ day of prednisone compared with patients not treated with corticosteroids. In the MSKCC cohort, the use of corticosteroids within 30 days before starting anti-PD(L)1 treatment was also collected. Interestingly, patients who were receiving corticosteroids within 30 days of initiating treatment with anti-PD-(L)1 but discontinued at some point before receiving the first dose had an intermediate survival outcome compared with patients who did not receive any corticosteroids at all. Nevertheless, specific information on the length of steroid use or days from steroid discontinuation to ICI initiation is lacking. The retrospective design of the study and the relatively small number of patients included in the corticosteroid subgroup are major limitations of the study. Indeed, as patients receiving corticosteroids are more likely to have unfavorable prognostic factors, such as poor ECOG PS, brain metastases, high tumor burden, or significant comorbidities, it is difficult to distinguish the potential negative impact of steroids from the intrinsic poor prognosis of the individuals who require steroids. Another limitation of the study is that patients were not stratified according to the indication for corticosteroids, probably due to the small number of patients. Different reasons for using steroids may have an impact on prognosis, especially if patients are taking steroids to overcome comorbidity exacerbations or to alleviate cancer-related symptoms.

Two additional studies have also reported an unfavorable effect of corticosteroids in patients with advanced NSCLC treated with ICI, and are summarized in Table $1(3,5)$. In both studies, use of corticosteroids at baseline or at ICI initiation consistently correlated with worse outcome in terms of PFS and OS. However, there were differences in the study population and in the time period to assess corticosteroid use. In both studies, the multivariate analysis showed a detrimental effect of corticosteroids independently of other clinical covariates (ECOG PS, age, presence of brain metastases).

Strikingly, when corticosteroids or other immunomodulating agents, such as anti-TNF therapy, have been used to treat immune related adverse events (irAEs) associated with ipilimumab treatment in patients with melanoma there was not an unfavorable effect on OS or time to treatment failure (6). In this sense, the impact of corticosteroids used to manage irAEs on the clinical outcome of patients with advanced NSCLC was assessed in two post-hoc analyses from the KEYNOTE-001 and OAK clinical trials $(7,8)$. Both analyses showed that patients with NSCLC receiving corticosteroids to manage irAEs did not experience worse overall response rate, PFS or OS. Since the number of patients receiving corticosteroids was small $(n=28$ in the KEYNOTE-001 and $n=24$ in the OAK trial), confirmation of these observations is warranted in prospective studies.

Information regarding CTLA-4 blockade and use of corticosteroids is scarce in NSCLC patients. Although using corticosteroids for the management of irAEs due to CTLA4 blockade has not been shown to have a detrimental effect in treatment efficacy (6), the impact of baseline high-dose corticosteroids prior to CTLA-4 blockade has not been investigated in the clinic. Preclinical data suggest that corticosteroids may have implications on CTLA-4 blocking antibodies like ipilimumab. Dexamethasone blocks naïve 


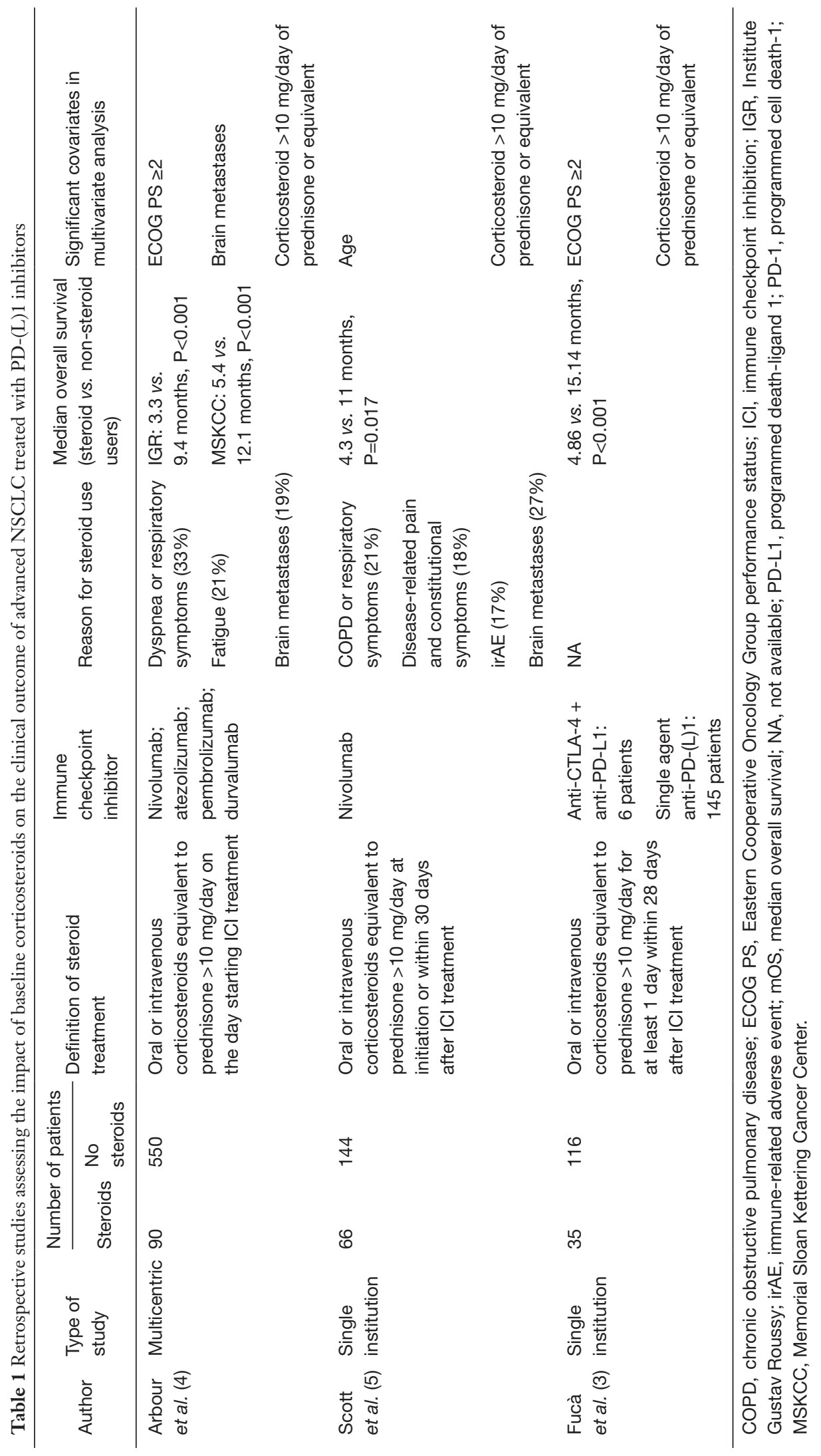


T-cell proliferation and differentiation by attenuating CD28 co-stimulation, while upregulating CTLA-4 expression in $\mathrm{CD}^{+}$and $\mathrm{CD}^{+} \mathrm{T}$ cells. CTLA-4 blockade can partially rescue $\mathrm{T}$ cells from the immunosuppressive effects of dexamethasone (9). CTLA-4, but not PD(L)-1 blockade can partially prevent the inhibitory effects of dexamethasone on the immune response.

Clinical trials assessing the combination of PD-(L) 1 blockade plus chemotherapy as first-line treatment for advanced NSCLC also excluded patients who were receiving corticosteroids at the time of randomization. However, corticosteroids are also part of most chemotherapy regimens and most phase III chemotherapy-ICI combination clinical trials in the first line setting have been positive (10-12). In this regard, a post-hoc analysis conducted in the KEYNOTE-407 study showed similar efficacy results for patients receiving the nab-paclitaxel regimen, which allows to reduce the dose of corticosteroids, compared with patients who received the paclitaxel regimen (13). These results suggest that short courses of corticosteroids might have a small impact on the immune function and clinical outcome.

In conclusion, the work of Arbour et al. is relevant because use of high dose corticosteroids at baseline or shortly after starting single PD-(L)1 blockade appears to have a negative impact on clinical outcome in patients with advanced NSCLC (4). However, the results from current retrospective studies do not allow to discern whether corticosteroids have unfavorable predictive value for ICI blockade or are just reflecting a subgroup of poor-risk patients with dismal prognosis. In other words, it is difficult to know whether corticosteroid use is a prognostic factor rather than a predictive factor of poor outcome. To address this issue, longitudinal studies should be conducted to prospectively collect the timing and dosing (pre- and postICI) of corticosteroids and their indication, as well as to characterize patient comorbidities by using validated scales like the Charlson comorbidity index, simplified comorbidity score, or cumulative illness rating scale (CIRS) (14-16). For now, caution is recommended when using corticosteroids prior to $\mathrm{PD}-(\mathrm{L}) 1$ blockade, since they can impair the ability of the immune system to attack tumor cells and may lessen the efficacy of immunotherapy.

\section{Acknowledgments}

Funding: E Nadal received support from the SLT006/17/00127 grant, funded by the Department of Health of the Generalitat de Catalunya by the call "Acció instrumental d'intensificació de professionals de la salut" and the PROYBAR17005NADA project funded by the AECC Barcelona (Spanish Association Against Cancer Barcelona). We thank CERCA Program/ Generalitat de Catalunya for their institutional support and grant 2017SGR448. M Jové is supported by a Rio Hortega contract (CM17/00008) from the Carlos III Institute. Copyediting editorial support was provided by Aurora O'Brate.

\section{Footnote}

Conflicts of Interest: Ernest Nadal received consulting honoraria from MSD, BMS, Roche and AstraZeneca.The other authors have no conflicts of interest to declare.

Ethical Statement: The authors are accountable for all aspects of the work in ensuring that questions related to the accuracy or integrity of any part of the work are appropriately investigated and resolved.

\section{References}

1. Planchard D, Popat S, Kerr K, et al. Metastatic non-small cell lung cancer: ESMO Clinical Practice Guidelines for diagnosis, treatment and follow-up. Ann Oncol 2018;29:iv192-237.

2. Cain DW, Cidlowski JA. Immune regulation by glucocorticoids. Nat Rev Immunol 2017;17:233-47.

3. Fucà $G$, Galli $G$, Poggi $M$, et al. Modulation of peripheral blood immune cells by early use of steroids and its association with clinical outcomes in patients with metastatic non-small cell lung cancer treated with immune checkpoint inhibitors. ESMO Open 2019;4:e000457.

4. Arbour KC, Mezquita L, Long N, et al. Impact of Baseline Steroids on Efficacy of Programmed Cell Death-1 and Programmed Death-Ligand 1 Blockade in Patients With Non-Small-Cell Lung Cancer. J Clin Oncol 2018;36:2872-8.

5. Scott SC, Pennell NA. Early Use of Systemic Corticosteroids in Patients with Advanced NSCLC Treated with Nivolumab. J Thorac Oncol 2018;13:1771-5.

6. Horvat TZ, Adel NG, Dang TO, et al. Immune-Related Adverse Events, Need for Systemic Immunosuppression, and Effects on Survival and Time to Treatment Failure in Patients With Melanoma Treated With Ipilimumab at Memorial Sloan Kettering Cancer Center. J Clin Oncol 2015;33:3193-8.

7. Leighl N, Gandhi L, Hellmann MD, et al. Pembrolizumab for NSCLC: Immune-mediated adverse events and 
corticosteroid use. J Thorac Oncol 2015;10:S233.

8. von Pawel J, Syrigos K, Mazieres J, et al. Association between immune-related adverse events (irAEs) and atezolizumab efficacy in advanced NSCLC: analyses from the phase III study OAK. Ann Oncol 2017;28: abstr 1314P.

9. Giles AJ, Hutchinson MND, Sonnemann HM, et al. Dexamethasone-induced immunosuppression: mechanisms and implications for immunotherapy. J Immunother Cancer 2018;6:51.

10. Gandhi L, Rodríguez-Abreu D, Gadgeel S, et al. Pembrolizumab plus Chemotherapy in Metastatic NonSmall-Cell Lung Cancer. N Engl J Med 2018;378:2078-92.

11. Paz-Ares L, Luft A, Vicente D, et al. Pembrolizumab plus Chemotherapy for Squamous Non-Small-Cell Lung Cancer. N Engl J Med 2018;379:2040-51.

12. Socinski MA, Jotte RM, Cappuzzo F, et al. Atezolizumab

Cite this article as: Jove M, Vilariño N, Nadal E. Impact of baseline steroids on efficacy of programmed cell death-1 (PD-1) and programmed death-ligand 1 (PD-L1) blockade in patients with advanced non-small cell lung cancer. Transl Lung Cancer Res 2019;8(Suppl 4):S364-S368. doi: 10.21037/tlcr.2019.06.06 for First-Line Treatment of Metastatic Nonsquamous NSCLC. N Engl J Med 2018;378:2288-301.

13. Halmos B, Luft A, Majem M, et al. MA10.08 Choice of Taxane and Outcomes in the KEYNOTE-407 Study of Pembrolizumab Plus Chemotherapy for Metastatic Squamous NSCLC. J Thorac Oncol 2018;13:S391.

14. Charlson ME, Pompei P, Ales KL, et al. A new method of classifying prognostic comorbidity in longitudinal studies: development and validation. J Chronic Dis 1987;40:373-83.

15. Colinet B, Jacot W, Bertrand D, et al. A new simplified comorbidity score as a prognostic factor in non-small-cell lung cancer patients: description and comparison with the Charlson's index. Br J Cancer 2005;93:1098-105.

16. Linn BS, Linn MW, Gurel L. Cumulative illness rating scale. J Am Geriatr Soc 1968;16:622-6. 